\title{
A Specific Component of the Evoked Potential Mirrors Phasic Dopamine Neuron Activity during Conditioning
}

\author{
Wei-Xing Pan and $\odot$ Joshua T. Dudman \\ Janelia Research Campus, Howard Hughes Medical Institute, Ashburn, Virginia 20147
}

Midbrain dopamine (DA) neurons are thought to be a critical node in the circuitry that mediates reward learning. DA neurons receive diverse inputs from regions distributed throughout the neuraxis from frontal neocortex to the mesencephalon. While a great deal is known about changes in the activity of individual DA neurons during learning, much less is known about the functional changes in the microcircuits in which DA neurons are embedded. Here we used local field potentials recorded from the midbrain of behaving mice to show that the midbrain evoked potential $(\mathrm{mEP})$ faithfully reflects the temporal and spatial structure of the phasic response of midbrain neuron populations during conditioning. By comparing the mEP to simultaneously recorded single units, we identified specific components of the mEP that corresponded to phasic DA and non-DA responses to salient stimuli. The DA component of the mEP emerged with the acquisition of a conditioned stimulus, was extinguished following changes in reinforcement contingency, and could be inhibited by pharmacological manipulations that attenuate the phasic responses of DA neurons. In contrast to single-unit recordings, the $\mathrm{mEP}$ permitted relatively dense sampling of the midbrain circuit during conditioning and thus could be used to reveal the spatiotemporal structure of multiple intermingled midbrain circuits. Finally, the mEP response was stable for months and thus provides a new approach to study long-term changes in the organization of ventral midbrain microcircuits during learning.

Key words: associative learning; conditioning; dopamine; electrophysiology; evoked potentials; single-unit recording

Significance Statement

Neurons that synthesize and release the neurotransmitter dopamine play a critical role in voluntary reward-seeking behavior. Much of our insight into the function of dopamine neurons comes from recordings of individual cells in behaving animals; however, it is notoriously difficult to record from dopamine neurons due to their sparsity and depth, as well as the presence of intermingled non-dopaminergic neurons. Here we show that much of the information that can be learned from recordings of individual dopamine and non-dopamine neurons is also revealed by changes in specific components of the local field potential. This technique provides an accessible measurement that could prove critical to our burgeoning understanding of the molecular, functional, and anatomical diversity of neuron populations in the midbrain.

\section{Introduction}

Although the neuron is the fundamental unit of the CNS, critical functions have been ascribed to the emergent properties of ensembles of coactivated neurons (Buzsáki, 2010; Einevoll et al., 2013). Local field potentials (LFPs) reflect the summed contribu-

\footnotetext{
Received Oct. 3, 2014; revised May 29, 2015; accepted June 10, 2015.

Author contributions: W.-X.P. and J.T.D. designed research; W.-X.P. performed research; J.T.D. contributed unpublished reagents/analytic tools; W.-X.P. and J.T.D. analyzed data; W.-X.P. and J.T.D. wrote the paper.

All funding was provided by Howard Hughes Medical Institute. We thank Shared Resources at Janelia Research Campus for excellent technical support, notably Amy Hu, who provided histology support for this project. We also thank our colleagues at Janelia for helpful comments and discussion of this manuscript.

J.T.D. is a Group Leader at the Janelia Research Campus of the Howard Hughes Medical Institute. The authors declare no competing financial interests.

Correspondence should be addressed to Joshua T. Dudman, Howard Hughes Medical Institute, Janelia Research Campus, 19700 Helix Drive, Ashburn, VA 20147. E-mail: dudmanj@janelia.hhmi.org.

DOI:10.1523/JNEUROSCI.4096-14.2015

Copyright $\odot 2015$ the authors $\quad 0270-6474 / 15 / 3510451-09 \$ 15.00 / 0$
}

tion of ionic conductances in nearby neurons, and are thought to arise from transmembrane currents mediated both by ligandgated receptors and voltage-gated ion channels (Reimann et al., 2013). Therefore, LFPs are thought to reflect the synchronous activation of neuronal populations and the synaptic input by which such coincident activation is recruited (Mitzdorf, 1985; Buzsáki, 2010). A number of studies have shown that changes in the properties of the LFPs are correlated with behavioral features such as exploration (Kemere et al., 2013), attention (Sundberg et al., 2012), volition (Scherberger et al., 2005), perception of complex stimuli (Einevoll et al., 2013), and learning (Rogan et al., 1997).

Appetitive conditioning is a process by which a previously neutral stimulus, the conditioned stimulus (CS), acquires motivational value through repeated association with a rewarding outcome such as sweetened water, which is referred to as the unconditioned stimulus (US; Schultz, 2006; Bromberg-Martin et 
al., 2010). Midbrain dopamine (DA) neurons are thought to be critical for appetitive conditioning (Schultz, 1997). Indeed, the phasic responses of DA neurons reflect key features of conditioning processes such as acquisition (Schultz, 1998; Pan et al., 2005), extinction (Pan et al., 2008), generalization (Mirenowicz and Schultz, 1996), blocking (Waelti et al., 2001), and conditioned inhibition (Tobler et al., 2003). However, the circuit mechanisms that determine the phasic response of DA neurons to salient stimuli remain poorly understood (Bromberg-Martin et al., 2010). This is due in large part to the notorious technical difficulty of measuring single DA neurons from behaving animals over sustained periods.

When recordings of the LFPs are aligned to the onset of a salient stimulus, it is common to observe a characteristic sequence of fluctuations in the amplitude of the LFPs-here described as evoked potentials (EPs; Mitzdorf, 1985). These stereotyped sequences of amplitude fluctuations in the EP are thought to reflect stages in the processing of sensory information and the initial recruitment of behavioral responses (Eggermont and Ponton, 2002). While many studies have examined the EPs recorded from superficial brain structures such as the cortex (Scherberger et al., 2005) and hippocampus (Múnera et al., 2001), and deep brain structures such as the amygdala (Quirk et al., 1995; Rogan et al., 1997; Rosenkranz and Grace, 2002), few descriptions of EPs in the ventral midbrain of the rodent exist [we refer to these measurements as the midbrain $\mathrm{EP}(\mathrm{mEP})]$. Despite the lack of such description, DA neurons have several characteristics that could yield reliable EPs — they are packed in a layer with partially aligned dendritic arbors (Gerfen, 2004) and are recruited transiently with a highly stereotyped latency $(\sim 50 \mathrm{~ms}$; Schultz, 2001). Thus, we reasoned that the mEP could shed light on the circuit mechanisms that underlie the recruitment of synchronous bursts of activity in neurons in response to salient stimuli.

However, the ventral midbrain is a diverse area with distinct, but tightly intermingled, neuronal subtypes. For example, we recently discovered that non-DA neurons in the midbrain show phasic responses to salient stimuli that exhibit learning dynamics that are similar to those of DA neurons (Pan et al., 2013). DA and non-DA neurons in the midbrain have characteristic, but distinct, latencies in their phasic responses to salient auditory stimuli (Pan et al., 2013). Here, we use simultaneous single-unit and LFP recordings to demonstrate that distinct temporal components of the mEP do indeed correspond to DA and non-DA single-unit responses. Moreover, one $\mathrm{mEP}$ component was selectively predictive of simultaneously recorded single-unit responses from DA neurons. This specific component exhibited learning-related changes in amplitude, and shared the stimulus specificity and pharmacological sensitivity of DA neurons. Finally, as a proof of principle, we show that the $\mathrm{mEP}$ can provide a dense measurement of the spatiotemporal organization of midbrain microcircuits.

\section{Materials and Methods}

Subjects and surgery. Six adult (average weight, 30 g; 3-6 months old) male mice (C57/Black6) were used. Mice were trained to learn a classic trace-conditioning task with a tone $(10 \mathrm{kHz}, 500 \mathrm{~ms}$ duration) as the CS and sweetened water as a reward (2000-2500 ms delay). Recording electrodes, 16- or 32-microwire arrays (http://www.inphysiology.com), were stereotaxically implanted under anesthesia (isoflurane $1.5 \%-2.5 \%$ in $\mathrm{O}_{2}$ ), aiming at the substantia nigra $(\mathrm{SN})$ and the ventral tegmental area (VTA) of the ventral midbrain (3.0-4.5 mm posterior to bregma; $0.5-2.0$ $\mathrm{mm}$ lateral to midline; and $3.5 \mathrm{~mm}$ for movable arrays or $4.5 \mathrm{~mm}$ for fixed arrays below the surface of skull). Animals were allowed at least 1 week for recovery from surgery and before undergoing any behavioral training or recording.

Animal care. All procedures and animal handling were performed in strict accordance with protocols (8-36 and 11-39) that were approved by the Institutional Animal Care and Use Committee and were consistent with the standards set forth by the Association for Assessment and Accreditation of Laboratory Animal Care. Mice were initially housed in a temperature- and humidity-controlled room maintained on a reversed $12 \mathrm{~h}$ light/dark cycle. Following 1 week of recovery from surgery, the water consumption of the mice was restricted to water consumed in the operant conditioning chamber. Mice underwent daily health checks, and water restriction was eased if mice fell to $<80 \%$ of their body weight at the beginning of water restriction. Mice were then familiarized with the training and recording box before behavioral training.

Behavioral training. Freely behaving mice were trained on an auditory trace-conditioning task with a tone $(10 \mathrm{kHz}, 500 \mathrm{~ms}$ duration) or lightemitting diode array used as a CS. A water reward, delivered via the gating of a solenoid valve, was given with a delay of $2.5 \mathrm{~s}$ (or $2 \mathrm{~s}$ in a small number of cases). The timing of trial events was controlled by custom-written software and hardware. The water reward could be obtained from a recessed water port in the wall of the behavioral box, and port entries were monitored with an infrared beam break. Small volumes $(\sim 0.01 \mathrm{ml})$ of sweet water $(0.005 \mathrm{M}$ saccharin solution) were delivered to the spout via a computer-controlled solenoid. The intertrial intervals were chosen randomly from a uniform distribution over the range of 15-40 s. Extinction training then involved exposing the animal to equivalent randomly timed repetitions of the previously conditioned CS, but without solenoid activation. Each conditioning session was performed for 40-100 trials, while the following extinction session was performed for 80-120 trials.

Electrophysiology. For all recordings reported here, we used $35 \mu \mathrm{m}$ tungsten electrodes arranged in groups of $16(2 \times 8)$ or $32(4 \times 8)$ microwire arrays with a $150 \mu \mathrm{m}$ spacing between wires in a row and 300 $\mu \mathrm{m}$ spacing between rows. In many cases, we found in post hoc histology that the tracks of electrodes remained well separated after implantation; however, we observed that wires could occasionally violate this separation. This was generally observed as nearly identical voltage signals, and in such cases only one of the identical recordings was used. Electrode arrays were either implanted at a fixed depth or attached to a customdesigned microdrive with similar results. Movable electrode arrays were advanced in 30-60 $\mu \mathrm{m}$ increments daily to search for independent units. A low impedance reference electrode was integrated into the array with a tip $1 \mathrm{~mm}$ shorter than the tips of recording electrodes. A 0.008 inch silver ground wire was connected to a stainless steel screw that was fixed into the head bone of the mouse as a ground. The voltage signals from electrodes were amplified and filtered with a sequential analog filter $(0.1-7.6$ $\mathrm{kHz}$ bandpass) and digital filter $(750-7.6 \mathrm{kHz}$ bandpass). Channels with detectable activity were digitized at $30 \mathrm{kHz}$ and thresholded on-line, and voltage segments (30-50 samples) were recorded to disk using the Cerebus Data Acquisition System from Blackrock Microsystems (http://www. blackrockmicro.com/). Spikes were reisolated off-line on the basis of wave shape using the Plexon Offline Sorter (http://www.plexon.com/ products/offline-sorter). Putative DA cells were classified according to the following criteria: (1) low firing rate $(<10 \mathrm{~Hz})$; (2) relatively broad action potential $(>1.2 \mathrm{~ms})$; (3) phasic CS responses with onset latencies of 40-60 ms; and (4) profound (>50\%) inhibition by the $\mathrm{D}_{2}$ receptor agonist quinpirole $(400 \mu \mathrm{g} / \mathrm{kg}$, s.c.; most putative DA cells were tested, but not all; Pan et al., 2013).

Pharmacology. Quinpirole (400 $\mu \mathrm{g} / \mathrm{kg}$, s.c) or 3-(2-carboxypiperazin4-yl)propyl-1-phosphonic acid (CPP; $10 \mathrm{mg} / \mathrm{kg}$, i.p.) were injected after an acquisition session ( $>50$ trials), and then the animals were put back into the task box and the CS tones were given at random intervals to probe the stimulus response. Animals were not collecting rewards due to the sedative effects of quinpirole (analyzed under conditions analogous to those for analyzing CPP).

Histology. To confirm the position of recording sites, mice were anesthetized (isoflurane, $>3 \%$ ) and perfused with PBS then paraformaldehyde ( $4 \% \mathrm{w} / \mathrm{v}$ in PBS). Brains were post-fixed for $>24 \mathrm{~h}$. The brains were then sectioned (50-100 $\mu \mathrm{m}$ thickness) using a vibrating microtome (VT1200; Leica Microsystems). The slices were imaged on a fluorescent mi- 


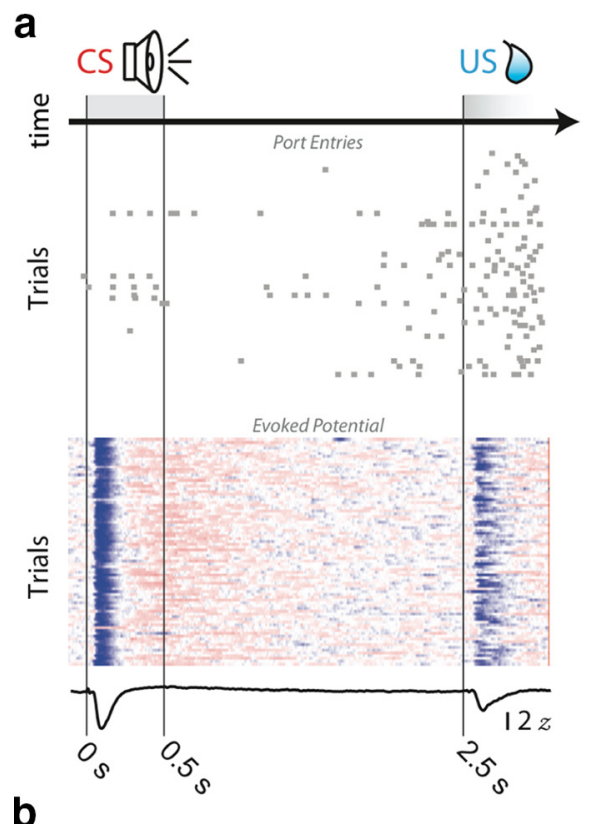

b
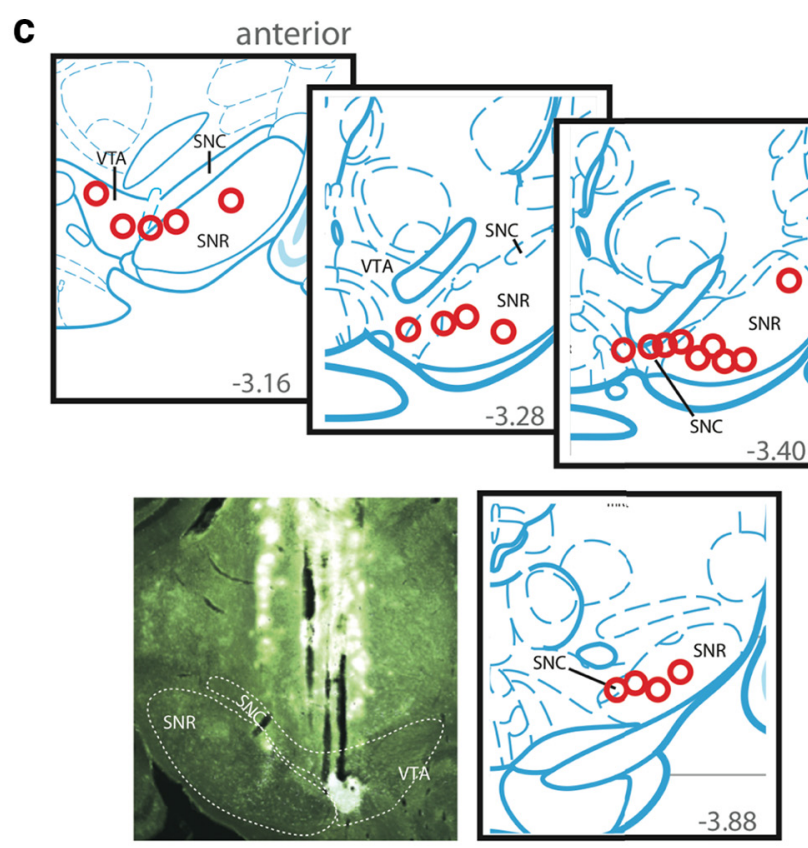
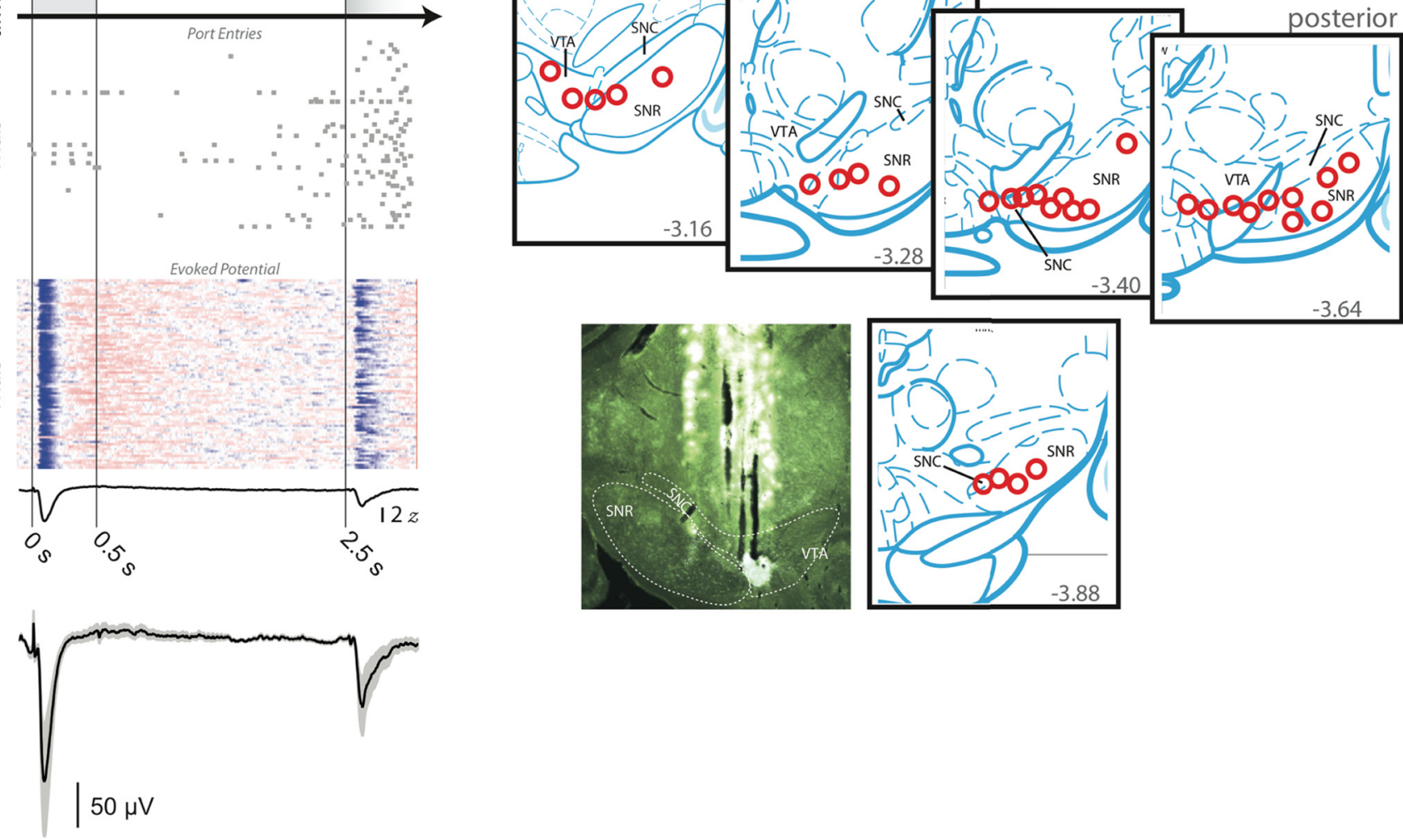

Figure 1. Description of the recording conditions, typical mEP observations, and task design. $\boldsymbol{a}$, Mice were placed in a behavioral chamber in which a pure tone ( $500 \mathrm{~ms}$ duration; $10 \mathrm{kHz}$; $\mathrm{CS}$ ) was delivered through a speaker followed by delivery of a water reward (US) $2 \mathrm{~s}$ after the end of the tone. Learning was assessed by the increase in entries to the water port following the onset of tone delivery. "Port entries," Entries to the water port are shown as a raster plot (middle) for an example session; "Evoked potential," a simultaneously recorded mEP is shown as a heat map plot [range, -2 (blue) to +2 (red) $z$-scored units]. The mean mEP response for the session is plotted as a solid black line. $\boldsymbol{b}$, The mean mEP recorded from the SN $\backslash \mathrm{VTA}$ area during acquisition across mice ( $n=$ 6). The shaded area indicates the SEM. $c$, The estimated anatomical position of recording sites for all successfully recovered electrode tracks was superimposed on the standard mouse brain atlas from Paxinos and Franklin (2004). Dopamine neuron-containing structures SNc, SNr, and VTA/PBP are labeled. Gray numbers indicate the position of the reference coronal slice relative to bregma (negative values are posterior). Inset, An example histological slice and corresponding atlas positions. Electrodes were coated with a hydrophobic fluorophore (http://www.lifetechnologies.com/ order/catalog/product/V22889?ICID = search-product) to aid in the tracing of electrode tracks through histological sections. Bright spot at the most ventral and medial electrode is the location of an electrolytic lesion used to identify the position of an electrode in which a prominent $(90$ component and putative dopamine neurons were recorded.

croscope, and the electrode tracks were mapped onto standard atlas sections by visual inspection.

Data analysis. The latencies and amplitudes of the mEPs or the firing rate of cells associated with task events were examined by constructing trial-by-trial raster displays and averaged pre-event time histograms (or wave form) using NeuroExplorer (http://www.neuroexplorer.com) and custom-written functions in Matlab version 2012b (http://www.mathworks.com/products/matlab/). The graphs of mEPs were routinely made with $1 \mathrm{~ms}$ bin widths for population averaging and statistical analysis. The peak latencies of a channel with the best wave form of each animal were measured with bin widths of $1 \mathrm{~ms}$, but $5 \mathrm{~ms}$ bins were used for statistical tests of the changes in amplitudes. The paired data of $100 \mathrm{~ms}$ before CS (onset) and $400 \mathrm{~ms}$ after CS during acquisition and extinction (or before and after drug injections) were analyzed with a $t$ test (paired, two-tailed). All statistical tests were performed using the Statistics Toolbox for Matlab (http://www.mathworks.com/help/stats/index.html) and are indicated in the text or figure legends. Physiological responses were defined as the peak firing rate in the first $200 \mathrm{~ms}$ after CS onset for single units or integration of the mEP waveform over the intervals 5-20 ms (C15), 20-40 ms (C30), and 55-150 ms (C90).

\section{Results}

Although the waveform of the $\mathrm{mEP}$ was variable across recording locations and mice, we found that three clear components were reliably observed across many recording sites (Fig. 1). We refer to these components by quantifying the latency until the peak deflection of the voltage, as follows: $\mathrm{C} 15$ [(mean $\pm \mathrm{SD}) 15.4 \pm 2.0 \mathrm{~ms}], \mathrm{C} 30$ $(28.6 \pm 4.8 \mathrm{~ms})$, and C90 (93.8 $\pm 5.9 \mathrm{~ms}$; see Fig. $2 a)$. The latencies of these characteristic components of the $\mathrm{mEP}$, while idiosyncratic across animals and recording sites, were apparent in the mean mEP. We found that $23 \%$ of electrodes implanted ( 22 of 96 electrodes) had a C90 component. Moreover, the latencies of the $\mathrm{mEP}$ components closely matched the distribution of latencies observed in a dataset of single-unit recordings that we described previously $(14.9 \pm 1.4$, $37.9 \pm 8.5$, and 83.2 \pm 18.4 ms; see Fig. $2 c$; Pan et al., 2013). In the dataset reported here, we replicate these results by observing simultaneously recorded single units with a similar distribution of response latencies (see Fig. $2 b, c$ ). We previously described these populations of individual units as GABA1, GABA2, and DA neurons, respectively, based upon firing properties, waveforms, optogenetic tagging, and pharmacological sensitivity (Pan et al., 2013). Thus, these data indicate that an auditory CS evokes a reliable sequence of activation of neuronal firing in the VTA/SN that is reflected as a reliable sequence of amplitude fluctuations in the $\mathrm{mEP}$. Specifically, the close correspondence in latencies in these data suggests that the C90 component of the mEP is a specific correlate of the phasic spiking response of DA neurons to salient stimuli. 
A specific component of the $\mathrm{mEP}$ reflects phasic dopamine neuron activity

The canonical features identified from single-unit recordings were observed in the C90 component of the mEP. (1) In addition to their characteristic latency, the phasic response of midbrain DA neurons is also more robust after a CS compared with a predicted US with extended training. Indeed, we found that both the C90 component of the $\mathrm{mEP}$ and simultaneously recorded DA units exhibited much smaller responses to a predicted US than to the CS (Figs. 1b, 2). (2) Both the C90 component of the mEP and the responses of simultaneously recorded DA units could be rapidly extinguished ( $T=2.63, n=15, p=0.019$, for the C90 amplitude; Fig. 3). In addition, consistent with previous work (Pan et al., 2013) showing that short-latency responses to the CS in non-DA neurons can be enhanced during extinction, we observed a significant enhancement of the C30 component of the mEP during extinction $(T=3.65, n=15$, $p=0.003$; Fig. $3 d$ ). However, there was no significant change in the $\mathrm{C} 15$ amplitude ( $T=0.2, n=15, p=0.84$ ). (3) Finally, phasic responses of DA neurons to salient stimuli are independent of the sensory modality used (Schultz, 2001). We found that both the $\mathrm{C} 90$ component of the $\mathrm{mEP}$ and the responses of simultaneously recorded DA units exhibited robust responses to visual stimuli when used as a CS (Fig. 4). As expected, the latencies of both the response of putative DA units and the $\mathrm{mEP}$ were delayed by $\sim 40 \mathrm{~ms}$ in response to visual stimuli-presumably due to the increased latency of peripheral processing. The $\mathrm{mEP}$ peak had a latency of 130 $\mathrm{ms}(n=6)$ for a visual CS, as opposed to $94 \mathrm{~ms}$ for an auditory CS. The firing rate of DA units peaked at a latency of $120 \mathrm{~ms}(n=13)$, as opposed to $86 \mathrm{~ms}$ for an auditory CS.

In addition to these characteristic response properties, DA neurons also exhibit characteristic pharmacological sensitivity. (1) Systemic administration of quinpirole, a $\mathrm{D}_{2}$ receptor agonist, suppressed both the $\mathrm{C} 90$ component of the $\mathrm{mEP}$, and the phasic and tonic activity of a simultaneously recorded DA unit (Fig. $5 a-d$ ). The amplitude of C90 decreased significantly $(T=2.67, n=6, p=$ 0.044). Importantly, the short latency components of C15 and C30 were not suppressed, but were significantly enhanced (C15: $T=$ 13.69, $n=6, p=4 \mathrm{e}-5$; C30: $T=5.02, n=6, p=0.004$; Fig. $5 a-d$ ). (2) Using an elegant transgenic strategy that eliminated functional NMDA receptor activation in DA neurons, previous work demonstrated that NMDA receptors are necessary for the highinstantaneous frequency, phasic responses of DA neurons (Zweifel et al., 2009). We likewise found that systemic blockade of NMDA receptors abolished both the phasic response of DA units and selectively eliminated the $\mathrm{C} 90$ component of the $\operatorname{mEP}(T=2.60, n=6$, $p=0.048$; Fig. $5 e-h$ ). Again, we found that neither simultaneously recorded putative GABAergic neurons nor the early components of the $\mathrm{mEP}$ were reduced by NMDA receptor blockade, but they were significantly enhanced (C15: $T=4.54, n=6, p=0.006$; C30: $T=$ $10.46, p=0.0001, n=6$; Fig. $5 e-h)$. Thus, together these data demonstrate that the $\mathrm{C} 90$ component of the $\mathrm{mEP}$ also matches the pharmacological profile of single-unit activity in midbrain DA neurons.
C

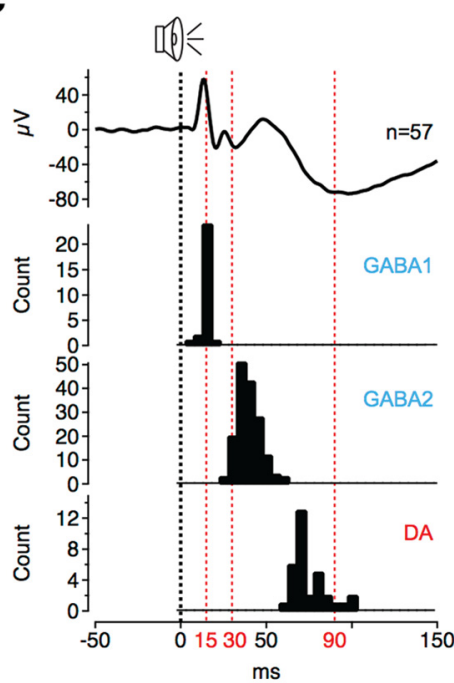

Figure 2. The components of the $\mathrm{mEP}$ of the midbrain elicited by auditory conditioned stimuli and single-unit activity recorded simultaneously. $\boldsymbol{a}$, Example mEP with multiple, clear components apparent across the population. Onset of the auditory CS mean $m E P$ across trials. Specific components are labeled as described in the text. $\boldsymbol{b}$ Three raster plots and corresponding peris(istograms for well isolated single units recorded simultaneously with the mEP. Two non-dopamine cells (top and The mean $m E P$ averaged across a number of electrodes and animals is aligned to the latency of the responses of a population of latency histogram for putative DA combines single units recorded previously (Pan et al., 2013) as well as 17 additional units not

It is important to note, however, that the site or the mechanism of effect, given the systemic administration of drug, could well be different for the mEP and single-unit responses. Thus, these data should be interpreted as providing evidence for the tight correlation between the $\mathrm{mEP}$ and single-unit activity across distinct pharmacological states of the animal.

In summary, the $\mathrm{mEP}$ provides a reliable correlate of the phasic activity of intermingled populations of midbrain neurons, including the previously identified short-latency activity of GABAergic neurons and the longer latency responses of units consistent with known features of DA neurons. We also observed that the $\mathrm{mEP}$ was a stable measurement in contrast to the relative difficulty of making stable recordings of single units. When we examined three mice with electrode arrays that were fixed in place (i.e., the $\mathrm{mEP}$ components were not changing due to electrode movement), we found that stable mEP components could be recorded on specific electrode months (Fig. 6; population data: three sites for 1.4 months, six sites for 4 months).

Evoked potentials can reveal the spatiotemporal organization of midbrain microcircuits

Separable components of the mEP reflect the precise activity patterns derived from specific subsets of single units isolated from the same sites. The SN/VTA is a complex anatomical structure that has multiple tortuous boundaries between cell types and afferent inputs. While single units from the same recording site can show diverse responses (e.g., DA and GABA units can be recorded on the same electrode), previous work from our group has suggested that the short latency responses to salient stimuli can be observed in GABAergic projection neurons of the SN pars reticulate $(\mathrm{SNr})$. Given that the $\mathrm{SNr}$ is spatially segregated from the densest populations of DA neurons in the SN pars compacata 

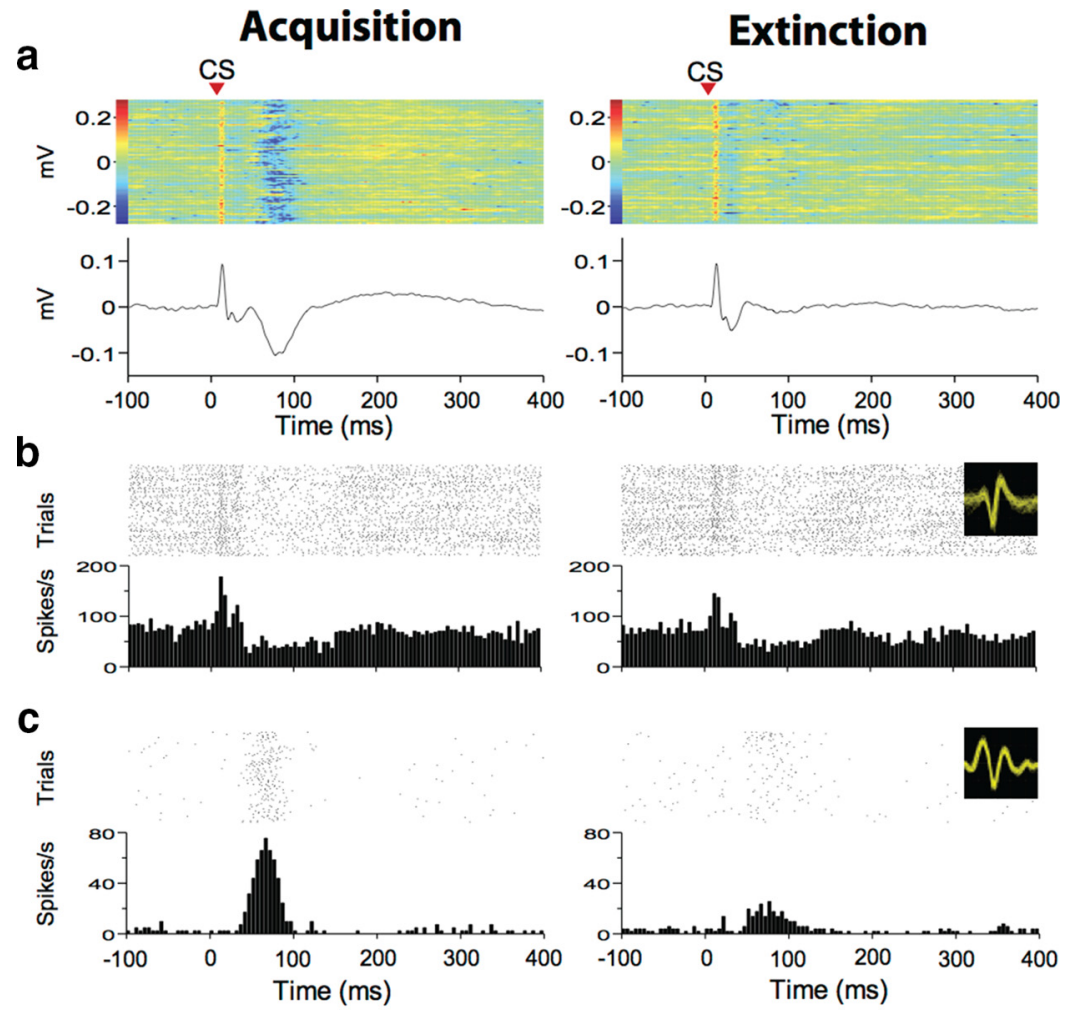

d

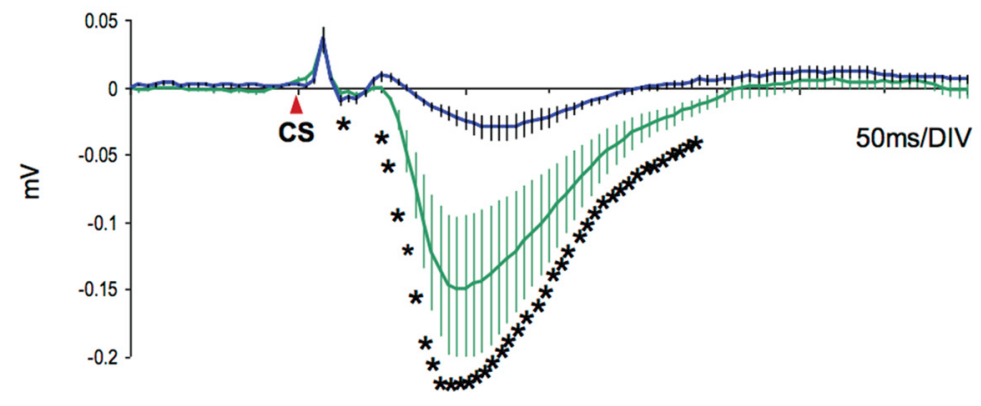

Figure 3. mEPs and single units recorded simultaneously during acquisition and extinction. $\boldsymbol{a}-\boldsymbol{c}$, An example session in which an $\mathrm{mEP}$ was recorded simultaneously with one non-DA and one putative DA single unit during acquisition (left) and extinction (right) of the auditory trace conditioning task. $\boldsymbol{a}$, Heat map plot in which each row is a trial. The lower plot shows the mean $\mathrm{mEP}$ response. CS onset is indicated by the red arrowhead. $\boldsymbol{b}, \boldsymbol{c}$, Raster plots and PSTHs for two example single units, one of which is a putative non-DA unit $(\boldsymbol{b})$ and one of which is a putative DA unit (c). Example waveforms for each sorted unit are shown in the inset plots. $\boldsymbol{d}$, Population data showing the average $\mathrm{mEP}$ recorded during acquisition (green) and extinction (blue) from 15 recording sites in five mice. Error bars reflect the SEM. Each asterisk indicates a time point at which a significant difference between acquisition and extinction was observed ( $p<0.05$, paired $t$ test).

(SNc) and VTA, this would suggest that a spatial gradient in $\mathrm{mEP}$ components could reflect the diverse spatiotemporal organization of midbrain responses to salient stimuli. To examine this possibility, we examined data from three mice in which a 32channel electrode array was implanted in the SN/VTA, and multiple single units were isolated from electrodes throughout the array and compared with the $\mathrm{mEP}$ recorded at the same sites.

Figure 7 shows a reconstructed estimate of the position of the electrode array relative to the SN/VTA of an example mouse in which 43 single units, distributed across the electrode array, were isolated in parallel with mEP recordings (Fig. 7a). Putative DA units were isolated and found to have canonical response properties (Schultz, 2001), including a robust phasic burst of activity centered at $\sim 90 \mathrm{~ms}$ after the onset of an auditory CS. This phasic response was robustly attenuated over the course of $\sim 100$ trials of extinction (Fig. $7 b$ ). Examining the spatial organization of elec- trode sites revealed a spatial clustering of sites with significant learning-related modulation of either the $\mathrm{C} 15 / \mathrm{C} 30$ or C90 components of the mEP (Fig. 7c). Notably, we found that electrodes with large C90 components that were larger in magnitude during acquisition were localized to relatively anterior and medial recording sites that are consistent with the anatomical locations of the medial SNc and VTA. By contrast, recording sites located relatively lateral and posterior, consistent with the anatomical location of the $\mathrm{SNr}$, were characterized by a prominent C15/C30 component, the magnitude of which increased during extinction. In general, we found that electrodes with significantly modulated $\mathrm{C} 15 / \mathrm{C} 30$ and C90 components were associated with the presence of single units with short-latency CS responses and putative DA units, respectively (Fig. 7c).

\section{Discussion}

Extracellular potentials measured either with electroencephalograms or electrical recordings from within the brain have long been used to study early time steps in the processing of sensory stimuli and cognition (Joos et al., 2014). A merit of EPs is their ability to provide the high temporal resolution that is necessary to observe stages of processing characterized by phasic, reliable activity and brief synaptic delays. However, the underlying neural activity that produces an EP can, in most cases, only be interpreted indirectly (Eggermont and Ponton, 2002). As a result of this limitation, the behavioral correlates of specific EP components have been examined more often. Here we use simultaneous single-unit and local field potential recordings to show, for the first time, that specific components of EPs recorded in the SN/VTA of the ventral midbrain from freely behaving mice, or mEPs, provide a precise correlate of the phasic responses of DA and non-DA neurons during associative learning. This provides a method that can simultaneously sample space and time with good resolution and thus may provide a critical tool for unraveling the spatiotemporal organization of microcircuits in the ventral midbrain.

Additionally, we suggest that mEPs may reveal heterogeneity in the underlying neural signals that can be difficult to observe from single-unit recordings alone-especially when strong signal correlations exist.

The mEP is a complex waveform that has distinct components at latencies between 15 and $90 \mathrm{~ms}$ after the onset of a salient stimulus. These latencies match the latencies of distinct populations of single units in the VTA/SN. The C30 and C90 components showed dramatic learning-dependent plasticity, which emerged and developed with an acquisition process of conditioning, and could be extinguished by extinction training. Thus, the 
a



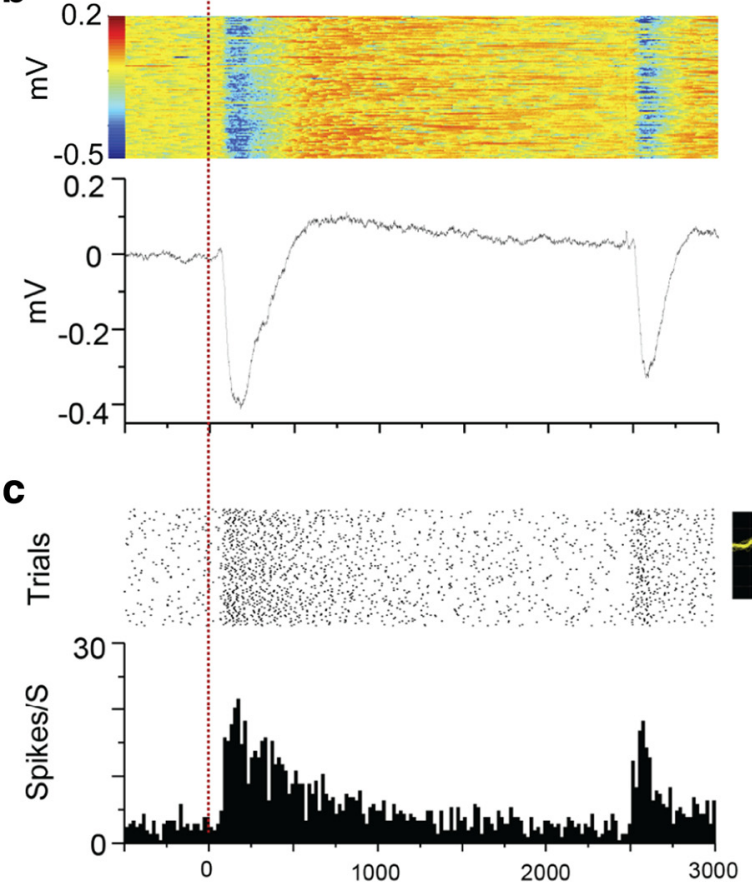

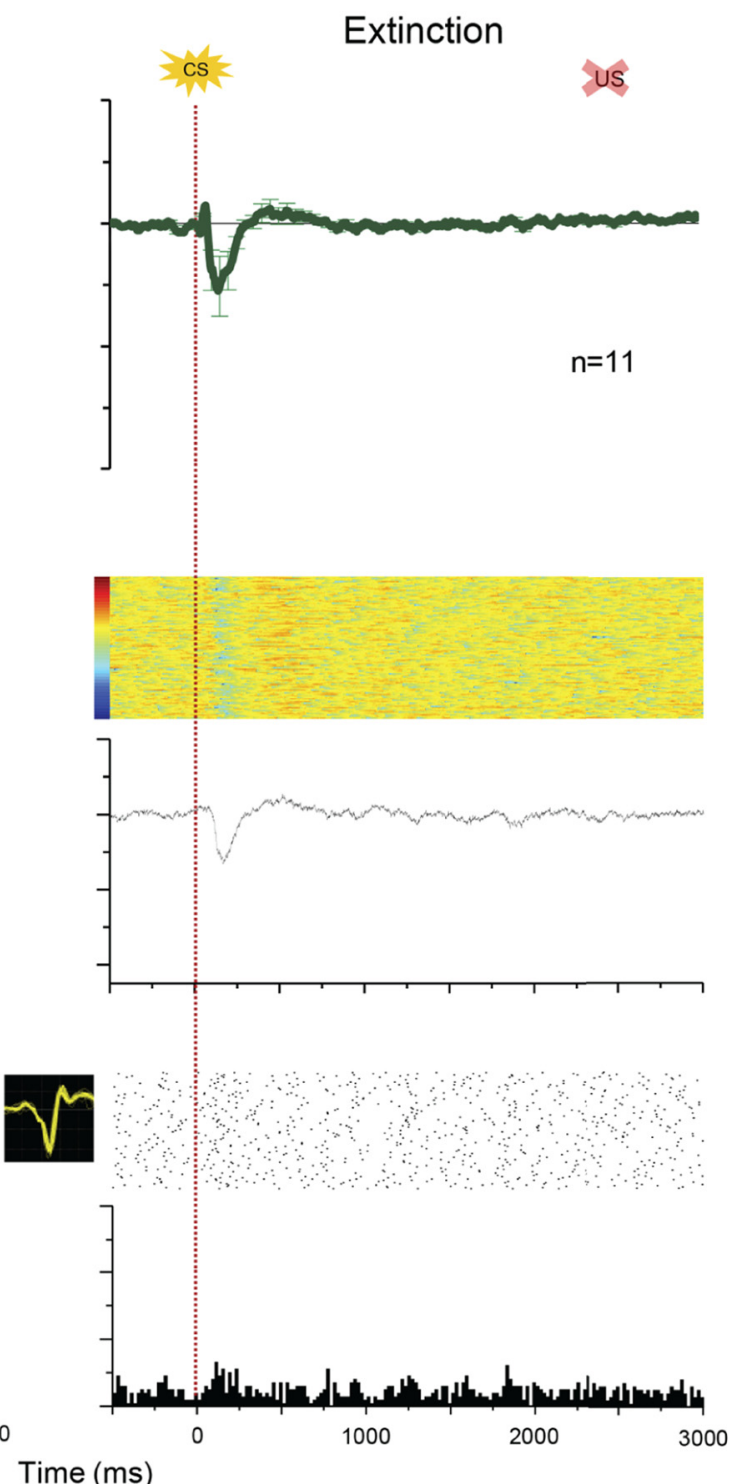

Figure 4. The responses to a visual CS during the acquisition and extinction. Recordings from the VTA/SN exhibit analogous responses to visual and auditory CSs during both acquisition (left) and extinction (right). $\boldsymbol{a}$, The population of $\mathrm{mEP}$ evoked by visual $\mathrm{CS}$ from 11 electrodes in two mice during acquisition and extinction. Error bars reflect the SEM. $\boldsymbol{b}$, $\boldsymbol{c}$, Example recordings of the mEP ( $\boldsymbol{b}$ ) and simultaneous putative DA single unit (c) during acquisition (left) and extinction (right). $\boldsymbol{b}$, Heat map plot of the mEP voltage for all trials aligned to CS onset. Average mEP shown below. $\boldsymbol{c}$, Raster plot (top) and PSTH (bottom) example of a single putative DA unit recorded on the same electrode as the mEP in $\boldsymbol{b}$ and aligned to the onset of the visual CS. Inset shows overlaid waveforms from the isolated single unit.

C30 and C90 reflect conditioned responses. The results from simultaneous recording with single-unit activity further suggested that $\mathrm{C} 90$ of the mEPs reflects phasic activity in DA neurons. We conclude this for the following reasons: (1) the latency of C90 matched the latency of phasic activation of DA cells; (2) the plasticity of C90 amplitude with conditioning processes of acquisition and extinction changed in parallel with the phasic firings of DA neurons; (3) the response to a predicted stimulus (in this case, a US) was diminished relative to the response to an unpredicted stimulus (in this case, a CS); (4) like DA neuron firing, C90 was elicited by conditioned stimuli independent of the sensory modality (with a longer latency to visual CS, which also matched the latency of DA neuron firing); (5) a shared pharmacological profile between C90 amplitude and single-unit firings of DA cells; and (6) single DA units were generally observed on channels in which a prominent $\mathrm{C} 90$ component with significant modulation by reward contingency was present.
In addition to the main findings cited above, we recorded at least two other components (C15 and $\mathrm{C} 30)$ of the mEP with shorter response latencies. The latencies of the $\mathrm{C} 15$ and $\mathrm{C} 30 \mathrm{com}-$ ponents were consistent with the latencies of GABA1 and GABA2 neurons in this area, as we have reported previously (Pan et al., 2013). Both GABA1 and GABA2 populations consist of some "extinction cells," which increase phasic activity to a CS selectively during extinction. We expected that the C15 and C30 could mirror the extinction process. However, C15 had stable peak latencies, and little change in amplitude between acquisition and extinction, suggesting that it may reflect a primarily sensory response, as opposed to a conditioned response. C30 showed an increase in amplitude during extinction, suggesting that C30 included the activity of GABA2 extinction cells. The C15 and C30 both increased modestly after the inactivation of DA neurons by quinpirole, which is further consistent with the modest increases in the spiking of non-DA neurons previously 




b

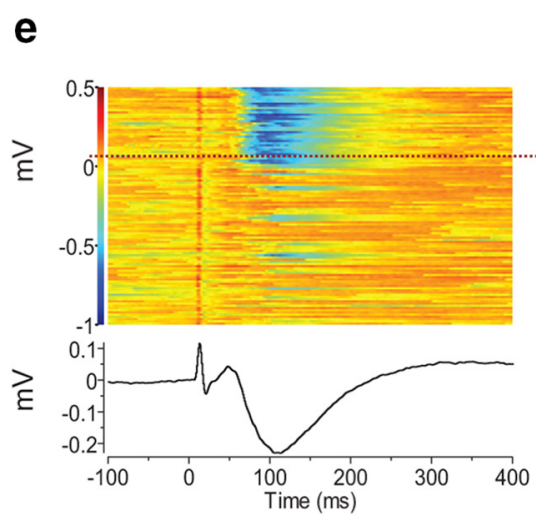

f

$\mathbf{f}$
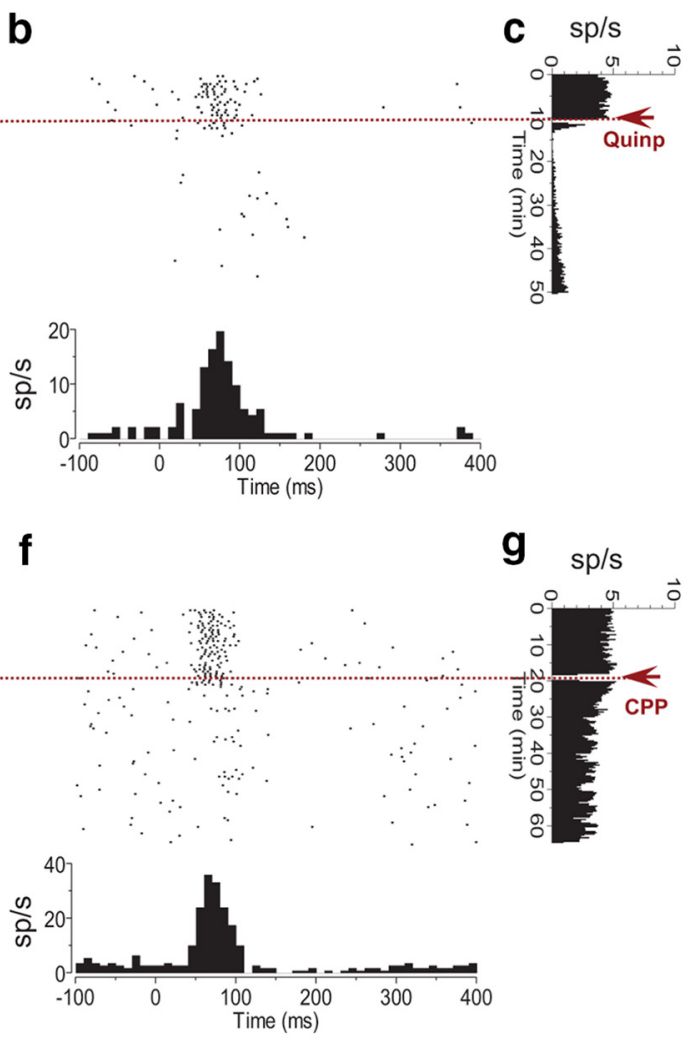

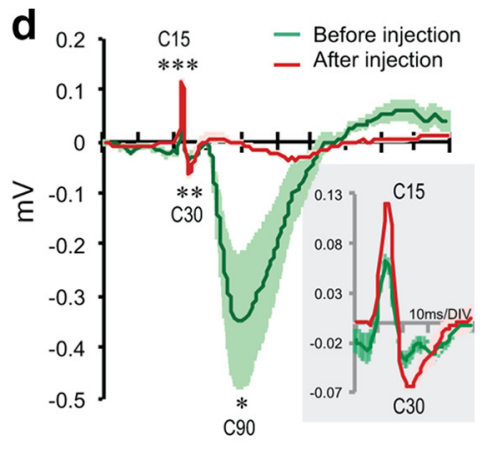

h

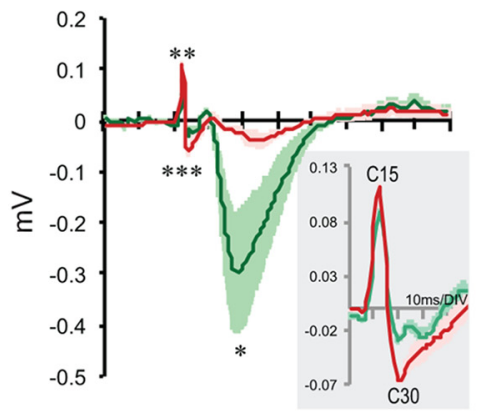

Figure 5. Shared pharmacology of $m E P$ and single dopamine units. $\boldsymbol{a}-\boldsymbol{d}$, The $\mathrm{mEP}$ and a putative $\mathrm{DA}$ single unit recorded before and during systemic injection of a $\mathrm{D}_{2}$ receptor agonist $[$ quinpirole (Quinp)]. $\boldsymbol{a}$, A heat map plot of trialwise amplitude of the $\mathrm{mEP}$ before (above dashed red line) and after drug injection. $\boldsymbol{b}$, Trial-aligned, simultaneously recorded putative DA single unit. $\boldsymbol{c}$, A histogram reflecting the instantaneous firing rate of the single unit in $\boldsymbol{b}$ (bin size, $10 \mathrm{~ms}$ ). $\boldsymbol{d}$, Average mEP waveform before (green) and after (red) quinpirole administration for all sessions. Inset shows a zoomed-in plot of the early C15/C30 components that were significantly increased in magnitude. $\boldsymbol{e}-\boldsymbol{h}$, Data plotted as described above, except for systemic injection of an NMDA receptor antagonist (CPP). In addition to significant reductions in the amplitude of the $\left(90\right.$ component of the $\mathrm{mEP}$, both of the drugs increased the peak amplitudes of $\mathrm{C} 15 / \mathrm{C} 30$ components. $^{*} p<0.05$; ${ }^{* *} p<0.01$; ${ }^{* * *} p<0.001$, paired $t$ test. Shaded area indicates the SEM. sp, Spikes.

\section{Day 12}

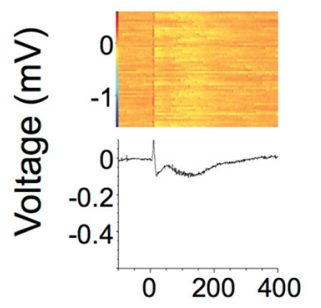

Time (ms)
Day 15

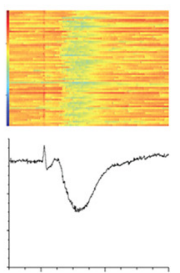

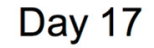
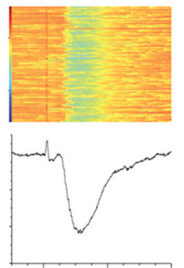
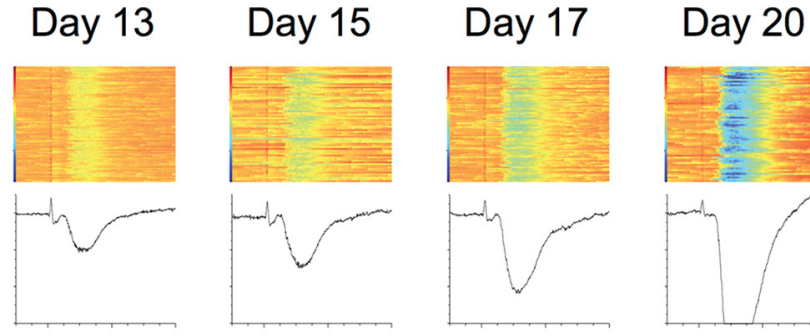
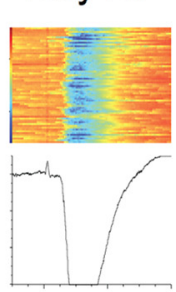

\section{0 days later}

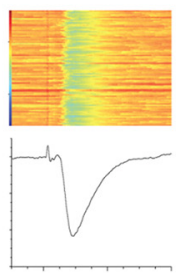

Figure 6. The ( 90 component of the mEP is stable for months. We observed that the 90 component of the mEP was stable for long periods. Across multiple mice, we found three sites with stable C 90 in amplitude and waveform for 1.4 months, and 6 sites stable for 4 months. An example recording of a 90 component stable for 4 months is shown. At this site, we found that a reliable mEP waveform appeared from day 12 of training and was stable throughout months of training. We note that there was a pause in training for several days before the "60 $\mathrm{d}$ later" time point was examined.

observed (Li et al., 2012). Together, these results suggest that the learning-related changes observed in non-DA neurons of the ventral midbrain are partially, but not entirely, reflected in the $\mathrm{mEP}$ (in particular, the GABA2 population). This may be due to postsynaptic changes in the GABA1 population that are not reflected in the $\mathrm{mEP}$ or may reflect a limitation in the measurement of the mEP.

We found that learning-related changes in the amplitude of $\mathrm{mEP}$ components could be used to reveal the spatially heterogeneous organization of microcircuits in the SN/VTA. Recent data have suggested that the DA neuron population is functionally heterogeneous, and reflects a combination of value, salience, and novelty signals (Lammel et al., 2008, 2011; Bromberg-Martin et al., 2010). Indeed, the stimulation of distinct inputs to DA neurons can produce opposing behavioral effects (Lammel et al., 2011). mEP recordings and the analysis techniques described herein could provide a complementary tool to further test or describe the functional heterogeneity in varied behavioral contexts. While there is substantial spatial overlap in populations of dopamine neurons with distinct connectivity, nonetheless, there is still partial segregation (Lammel et al., 2008). To record from these populations simultaneously would require dense spatial sampling of single DA units that is generally not possible. Thus, the $\mathrm{mEP}$ may provide an important alternative for sampling 


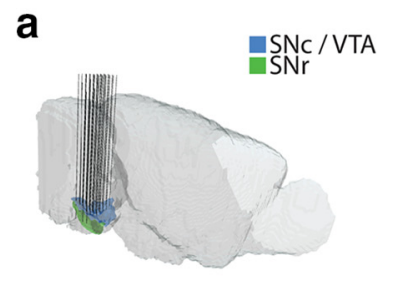

b

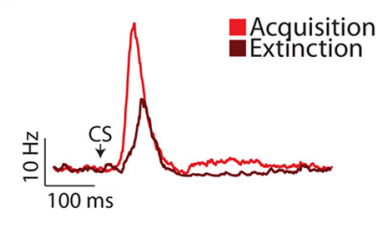

C
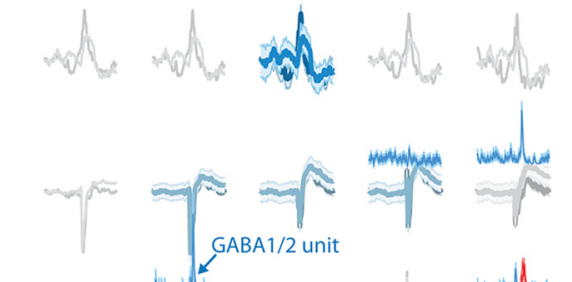
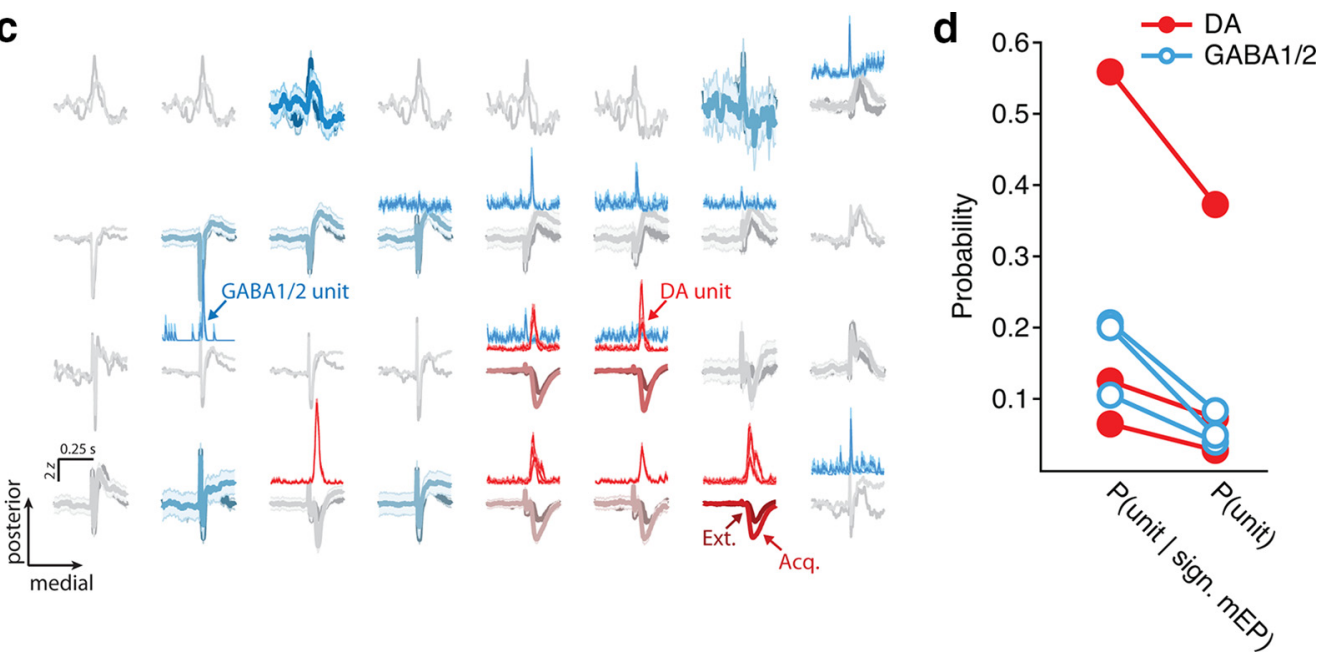

Figure 7. The spatial organization of single units is reflected in the mEP. We compared the spatial organization of the mEP for three mice in which 32 -channel arrays were implanted in the SN/VTA and multiple putative DA units were recorded simultaneously. $\boldsymbol{a}$, For a representative mouse, the position of the 32-channel array is shown with respect to the location of the SN/VTA. (Threedimensional mouse brain atlas was obtained from http://www.alleninstitute.org; the positions of the electrodes were estimated from tracing the tracts of individual wires in histological samples and a single electrolytic lesion). $\boldsymbol{b}$, Single dopamine neurons (the criteria for inclusion are described in Materials and Methods) exhibited large phasic modulations of firing rate that peaked $\sim 90 \mathrm{~ms}$ after the onset of a CS during acquisition (light red) of the auditory trace conditioning task. Subsequent extinction (repeated presentation of CS without reward; dark red) led to a significant reduction of the phasic response that was eventually completely attenuated with sustained extinction. (S onset is indicated by the black arrow. $c$, The average z-scored mEPs recorded on all 32 channels of the electrode array are shown for both acquisition (Acq.; lower traces; lighter colors) and extinction (Ext.; darker traces). CS onset occurs $0.25 \mathrm{sinto}$ the traces. Electrodes are arranged according to their physical position on the array with the top left position being the most posterior and lateral electrode. The thickness of the line in the mEP traces indicates sites with a significant change in the mEP between acquisition and extinction. The color of the traces indicates the magnitude of the change where decreases in the 90 component are indicated by the red saturation and increases in the C15/C30 components are indicated by cyan saturation. Thick gray mEP traces indicate sites with changes to multiple mEP components. The top traces are the $z$-scored peristimulus time histograms for putative DA (red) and GABA (cyan) neurons with long and short latencies ( $<60 \mathrm{~ms}$ ) after CS onset, respectively (Pan et al., 2013). [Note: peristimulus time histograms for DA units are plotted for acquisition trials; peristimulus time histograms for GABA units are plotted for extinction trials due to the increase in magnitude during extinction, as we reported previously (Pan et al., 2013)]. $\boldsymbol{d}$, Plot shows the contrast between the probability of detecting either a DA (red, filled) or GABA1/2 (cyan, open) unit on an electrode with learning-related C 90 and C15/C30 mEP components [P(unit / sign. $\mathrm{mEP})]$ to the overall frequency of $\mathrm{DA}$ and GABA1/2 units [P(unit)]. The analysis was derived from 166 total units recorded on 96 electrodes in three mice.

densely from the diverse populations of midbrain neurons. Consistent with this suggestion, we have demonstrated that two functionally distinct populations of single units in the midbrain, short-latency GABAergic neurons and long-latency putative dopamine neurons, tend to be recorded from spatially distinct recording sites. Likewise, large-amplitude C15/C30 and C30 mEP components with learning-related changes were observed on spatially segregated recording sites. The presence of these distinct $\mathrm{mEP}$ components was predictive of the response properties of single units recorded at the same sites and reflected the spatiotemporal organization of phasic responses to salient stimuli that were previously identified (Pan et al., 2013). Finally, we also found that, just as there are opponent changes in the phasic responses of these two populations to conditioned and extinguished stimuli, the sign of the change in the C15/C30 and C90 mEP components is likewise opposite. Together, these observations argue that the measurement of the $\mathrm{mEP}$ is sufficient to reveal both temporal and spatial aspects of the functional organization of midbrain circuits.

The biophysical basis of the extracellular potential has been a subject of debate for well over a century (Buzsáki et al., 2012). While there are some empirical data arguing that the LFP may be dominated by currents flowing through ligand-gated receptors at synapses, there is recent theoretical evidence that intrinsic, voltage-gated conductances may also contribute a substantial fraction of the observed potential changes, especially during strongly correlated spiking activity (Reimann et al., 2013). The phasic activity of DA neurons engages diverse intrinsic ion channels, but also depends upon transmembrane currents of NMDA receptors (Zweifel et al., 2009). If we assume this to apply to our measurements, then the mEP and spiking activity could be highly correlated during strong, relatively synchronized phasic input and the resultant postsynaptic spiking. However, a partial correlation could result from a few alternative mechanisms. For one, the input-output transformation of neurons can be nonlinear (either supralinear or sublinear) depending upon, for example, the specific subcellular distribution of synaptic inputs or the type of feedforward inhibition recruited. These nonlinearities could lead to differences between a signal that purely reflects spiking output (isolated single units) and a signal that also reflects synaptic input (mEPs). Thus, under such a circumstance, the mEP might be a more faithful report of circuit dynamics or convey distinct information about the input to the midbrain. For example, in the visual cortex, low-frequency components of the LFP can convey distinct information from simultaneously recorded single units (Belitski et al., 2008). On the other hand, a single neuron can have a large dendritic arbor that spans several recording sites of the mEP. Spiking activity that is detected perisomatically could show up on an electrode with no apparent $\mathrm{mEP}$ signal because the input that drove that spiking output originated hundreds of micrometers away. In this latter case, single-unit recordings could be thought of as providing a more faithful report of the response properties of cells with somata in that particular location. Thus, we expect that the utility of the $\mathrm{MEP}$ as a measure of the dynamics of activity in midbrain circuits could vary upon the specific question being addressed.

Here we show that the canonical properties of phasic DA and non-DA neuron activity derived from single-unit recordings were replicated by examining the temporal components of the $\mathrm{mEP}$. Unlike single-unit recordings, however, the $\mathrm{mEP}$ provides a method to record from a relatively even distribution over space. As a result, the $\mathrm{mEP}$ provides a unique measure of the spatiotem- 
poral organization of ventral midbrain circuits. As an example, here we confirm that the two opposing signals of acquisition and extinction that we had previously detected with single-unit recordings from distinct neuronal populations could both be detected with $\mathrm{mEP}$ recordings alone. Further, the relatively dense sampling possible with the $\mathrm{mEP}$ recordings revealed that these populations were partially intermingled, but with a clear spatial offset that was consistent with the short-latency response modulated by extinction that is derived in part from a population of GABAergic projection neurons in the SNr. In future applications, we anticipate that this measurement could prove critical to our burgeoning understanding of the molecular and anatomical diversity of neuron populations and the distinct microcircuits in which they are embedded.

\section{References}

Belitski A, Gretton A, Magri C, Murayama Y, Montemurro MA, Logothetis NK, Panzeri S (2008) Low-frequency local field potentials and spikes in primary visual cortex convey independent visual information. J Neurosci 28:5696-5709. CrossRef Medline

Bromberg-Martin ES, Matsumoto M, Hikosaka O (2010) Dopamine in motivational control: rewarding, aversive, and alerting. Neuron 68:815-834. CrossRef Medline

Buzsáki G (2010) Neural syntax: cell assemblies, synapsembles, and readers. Neuron 68:362-385. CrossRef Medline

Buzsáki G, Anastassiou CA, Koch C (2012) The origins of extracellular fields and currents-EEG, ECoG, LFP and spikes. Nat Rev Neurosci 13:407420. CrossRef

Eggermont JJ, Ponton CW (2002) The neurophysiology of auditory perception: from single units to evoked potentials. Audiol Neurootol 7:71-99. CrossRef Medline

Einevoll GT, Kayser C, Logothetis NK, Panzeri S (2013) Modelling and analysis of local field potentials for studying the function of cortical circuits. Nat Rev Neurosci 14:770-785. CrossRef Medline

Gerfen CR (2004) The basal ganglia. In: The rat nervous system, (Paxinos G, ed), pp 458-497. Amsterdam: Elsevier.

Joos K, Gilles A, Van de Heyning P, De Ridder D, Vanneste S (2014) From sensation to percept: the neural signature of auditory event-related potentials. Neurosci Biobehav Rev 42:148-156. CrossRef Medline

Kemere C, Carr MF, Karlsson MP, Frank LM (2013) Rapid and continuous modulation of hippocampal network state during exploration of new places. PLoS One 8:e73114. CrossRef Medline

Lammel S, Hetzel A, Häckel O, Jones I, Liss B, Roeper J (2008) Unique properties of mesoprefrontal neurons within a dual mesocorticolimbic dopamine system. Neuron 57:760-773. CrossRef Medline

Lammel S, Ion DI, Roeper J, Malenka RC (2011) Projection-specific modulation of dopamine neuron synapses by aversive and rewarding stimuli. Neuron 70:855-862. CrossRef Medline

Li W, Doyon WM, Dani JA (2012) Quantitative unit classification of ventral tegmental area neurons in vivo. J Neurophysiol 107:2808-2820. CrossRef Medline

Mirenowicz J, Schultz W (1996) Preferential activation of midbrain dopamine neurons by appetitive rather than aversive stimuli. Nature 379:449451. CrossRef Medline

Mitzdorf U (1985) Current source-density method and application in cat cerebral cortex: investigation of evoked potentials and EEG phenomena. Physiol Rev 65:37-100. Medline

Múnera A, Gruart A, Muñoz MD, Fernández-Mas R, Delgado-García JM (2001) Hippocampal pyramidal cell activity encodes conditioned stimulus predictive value during classical conditioning in alert cats. J Neurophysiol 86:2571-2582. Medline

Pan WX, Schmidt R, Wickens JR, Hyland BI (2005) Dopamine cells respond to predicted events during classical conditioning: evidence for eligibility traces in the reward-learning network. J Neurosci 25:6235-6242. CrossRef Medline

Pan WX, Schmidt R, Wickens JR, Hyland BI (2008) Tripartite mechanism of extinction suggested by dopamine neuron activity and temporal difference model. J Neurosci 28:9619-9631. CrossRef Medline

Pan WX, Brown J, Dudman JT (2013) Neural signals of extinction in the inhibitory microcircuit of the ventral midbrain. Nat Neurosci 16:71-78. CrossRef Medline

Paxinos G, Franklin KB (2004) The mouse brain in stereotaxic coordinates. San Diego: Academic.

Quirk GJ, Repa C, LeDoux JE (1995) Fear conditioning enhances shortlatency auditory responses of lateral amygdala neurons: parallel recordings in the freely behaving rat. Neuron 15:1029-1039. CrossRef Medline

Reimann MW, Anastassiou CA, Perin R, Hill SL, Markram H, Koch C (2013) A biophysically detailed model of neocortical local field potentials predicts the critical role of active membrane currents. Neuron 79:375-390. CrossRef Medline

Rogan MT, Stäubli UV, LeDoux JE (1997) Fear conditioning induces associative long-term potentiation in the amygdala. Nature 390:604-607. CrossRef Medline

Rosenkranz JA, Grace AA (2002) Dopamine-mediated modulation of odour-evoked amygdala potentials during pavlovian conditioning. Nature 417:282-287. CrossRef Medline

Scherberger H, Jarvis MR, Andersen RA (2005) Cortical local field potential encodes movement intentions in the posterior parietal cortex. Neuron 46:347-354. CrossRef Medline

Schultz W (1997) Dopamine neurons and their role in reward mechanisms. Curr Opin Neurobiol 7:191-197. CrossRef Medline

Schultz W (1998) Predictive reward signal of dopamine neurons. J Neurophysiol 80:1-27. Medline

Schultz W (2001) Reward signaling by dopamine neurons. Neuroscientist 7:293-302. CrossRef Medline

Schultz W (2006) Behavioral theories and the neurophysiology of reward. Annu Rev Psychol 57:87-115. CrossRef Medline

Sundberg KA, Mitchell JF, Gawne TJ, Reynolds JH (2012) Attention influences single unit and local field potential response latencies in visual cortical area V4. J Neurosci 32:16040-16050. CrossRef Medline

Tobler PN, Dickinson A, Schultz W (2003) Coding of predicted reward omission by dopamine neurons in a conditioned inhibition paradigm. J Neurosci 23:10402-10410. Medline

Waelti P, Dickinson A, Schultz W (2001) Dopamine responses comply with basic assumptions of formal learning theory. Nature 412:43-48. CrossRef Medline

Zweifel LS, Parker JG, Lobb CJ, Rainwater A, Wall VZ, Fadok JP, Darvas M, Kim MJ, Mizumori SJ, Paladini CA, Phillips PE, Palmiter RD (2009) Disruption of NMDAR-dependent burst firing by dopamine neurons provides selective assessment of phasic dopamine-dependent behavior. Proc Natl Acad Sci U S A 106:7281-7288. CrossRef Medline 